\title{
SEDENTARISMO EN LA POBLACIÓN DE 18 A 64 AÑOS DE LA CIUDAD DE PEREIRA, 2020
}

Luisa Fernanda Varona Vidal*, Carolina Herrera Quintero**, Jhonatan González Santamaría***, Claudia Jimena López García****

\section{Resumen}

Cuando se habla de conductas sedentarias, por lo general se habla de aquellas actividades que se realizan estando sentado, acostado o reclinado sin estar dormido. Los comportamientos sedentarios están asociados a enfermedades que generan grandes costos.

En la actualidad, en Pereira, no se cuenta con un registro actualizado que permita conocer la prevalencia de sedentarismo en la población adulta y en sí, en ningún momento del ciclo vital.

Estudio epidemiológico de prevalencia. En el cual la variable de estudio es el comportamiento sedentario en la población entre 18 a 64 años de edad del municipio de Pereira. La población de Pereira se tomó del DANE, en donde vivían 74.025 personas en el 2020. A partir de allí, se calculó una muestra representativa aleatoria de 950 personas, las cuales serán seleccionadas mediante técnicas de georreferenciación. Resultados esperados: generar información útil para la toma de decisiones en salud pública.

* Estudiante del programa de Fisioterapia, Fundación Universitaria del Área Andina. Correo: Ivarona@estudiantes.edu.co

** Estudiante del programa de Fisioterapia, Fundación Universitaria del Área Andina. Correo: cherrera30@estudiantes.edu.co

*** Docente programa de Fisioterapia, Fundación Universitaria del Área Andina. Correo: Jsantamaria13@areandina.edu.co

****Docente programa de Fisioterapia, Fundación Universitaria del Área Andina. Correo: clopez71@areandina.edu.co 


\section{Introducción}

La actividad física es reconocida como un factor fundamental para el cuidado de la salud individual y colectiva de los seres humanos. Desde 1985, Caspersen definió la actividad física como todo movimiento corporal humano en el cual se genera un gasto energético mayor al del reposo (1).

En la actualidad, se ha acumulado suficiente evidencia que demuestra una estrecha relación entre la actividad física que realizan las personas en el trabajo, el hogar, el tiempo libre y como medio de transporte. Es así que la actividad física deja de pensarse solo como algo que debe realizarse en todo momento de la vida (2), para garantizar así las mejores condiciones de salud metabólica y mental posible (3).

Ahora bien, a pesar de los múltiples beneficios que se han relacionado con la salud, la actividad física está lejos de ser el hábito más común en el grueso de la población mundial. Por el contrario, los comportamientos sedentarios se relacionan con un número de enfermedades que generan enormes costos a los sistemas de salud y a las economías de los hogares en todo el mundo (4).

Las formas de vida actual facilitan la adopción de hábitos de vida en donde los esfuerzos físicos son casi ausentes e innecesarios; esto ha conllevado la generación de nuevas categorías de investigación que permitan dar cuenta de los nuevos desafíos para la salud pública.

216 En la actualidad, en Colombia no se cuenta con un registro sistemático de las conduc- tas sedentarias y, por tanto, se desconoce la prevalencia de este factor de riesgo en los diferentes momentos del curso de vida.

Pensando en contribuir a satisfacer esta necesidad académica y de salud pública, se pretende desarrollar un estudio de prevalencia en donde la variable de estudio será el sedentarismo en la población adulta del municipio de Pereira.

\section{Planteamiento del problema}

Un comportamiento sedentario es aquella actividad que al realizarse genera un gasto calórico entre 1,0 y 1,5 MET (equivalentes metabólicos necesarios para realizar la actividad). Para la población adulta, estas actividades están asociadas a estar sentado, acostado o reclinado (5). El comportamiento sedentario es considerado un factor de riesgo para diversas enfermedades, principalmente para las no transmisibles de tipo metabólico (6). Son múltiples los estudios que reportan al sedentarismo como un factor de riesgo para diabetes, hipertensión, dislipidemias, demencia y otras enfermedades, independientemente del nivel de actividad física global de la persona.

Según reportes de la Organización Mundial de la Salud (OMS), la inactividad física y el comportamiento sedentario son factores de riesgo a nivel mundial. Una publicación de la prestigiosa revista Lancet en 2016, estimó que los costos asociados a la inactividad física ascendieron a 53,7 millones de dólares; incluso, se reportó que la inactividad física está asociada a la pérdida de 120000 millones de años por muerte y discapacidad prematura (7). Un metaanálisis de 
estudios epidemiológicos reportó una asociación de 1,46 entre el riesgo cardiovascular y los comportamientos sedentarios. La mortalidad por enfermedades cardiovasculares, cáncer y todas las grandes causas presentan una relación inversamente proporcional con los niveles moderados y vigorosos de la actividad física. Por otra parte, se ha demostrado una relación proporcional entre el riesgo de muerte por enfermedades metabólicas y el tiempo sentado medido. Consecuentemente, los estudios reportaron una relación proporcional entre el tiempo sentado y la mortalidad (8).

Diversos autores se manifiestan preocupados debido a la adopción de lo que se denomina el "estilo de vida occidental", el cual se caracteriza por bajos niveles de actividad física en el transporte y tiempo libre (9).

En el 2010, un estudio de prevalencia de actividad física en la ciudad de Pereira, reportó que cinco de cada 10 adultos cumplen con las recomendaciones mundiales de actividad física. Si bien, a partir de este estudio se pudo inferir la prevalencia de sedentarismo, no fue posible conocer el comportamiento de esta variable con relación a diversos factores sociodemográficos, que se hacen relevantes al momento de direccionar acciones desde las diferentes instituciones que se ocupan de la salud pública del municipio (10).

\section{Justificación}

Los comportamientos sedentarios tienen grandes implicaciones en la salud pública, se han convertido en un tema de interés que exige un abordaje y una transdisciplinaridad, para así entender cómo las complejas realidades de los grupos poblacionales facilitan o desestimulan la presencia de este fenómeno que hoy se ubica en los puntos más de morbimortalidad (11).

Colombia es un país diverso en vías de desarrollo, que está experimentando un creciente flujo de migrantes de lo rural a lo urbano. El estilo de vida urbano trae implícitos ciertos hábitos en los cuales el esfuerzo físico es poco necesario: automóviles, controles remotos, ascensores, telefonía celular, computadores, son algunas de las tecnologías que se han incorporado en el diario vivir citadino. Aunado a ello, las ciudades han crecido a un ritmo superior al que se pueden adaptar y la presión por viviendas suficientes y vías para los vehículos, han hecho que muchas ciudades sacrifiquen la cantidad de espacio público disponible para la práctica de la actividad física (12). Esto se convierte, claramente, en una barrera para quienes buscan incorporar en sus estilos de vida el movimiento corporal (13).

Según la Encuesta Nacional de Situación Nutricional (Ensin), versión 2015, el tiempo de actividad física se viene reduciendo desde el 2005 en la población adulta. En la actualidad, el 51,5\% de los adultos colombianos cumplen con las recomendaciones mundiales de actividad física promovidas por la OMS (14).

La Ensin es el único medio sistemático para el registro de los hábitos de vida relacionados con la salud con el que se cuenta en Colombia. Cabe resaltar que 
la Ensin se presenta con un intervalo de tiempo de siete años, periodo que no está alineado con los mandatos de los gobiernos locales y nacional.

Desde esta perspectiva, en el país no se cuenta con información actualizada y oportuna para que se haga seguimiento y evaluación al impacto de los programas de salud pública. Para la comunidad científica y el gobierno local de la ciudad, puede ser de gran ayuda contar con información accesible que dimensione la magnitud del sedentarismo como fenómeno de interés colectivo.

\section{Objetivos}

Objetivo general: determinar la prevalencia de sedentarismo en la población entre 18 y 64 años de edad de la ciudad de Pereira, 2020.

\section{Objetivos específicos:}

- Determinar el tiempo sentado de la población entre 18 a 64 años de la ciudad de Pereira.

- Identificar el nivel de actividad física de los adultos entre 18 y 64 años de la ciudad de Pereira.

\section{Referente teórico}

El término sedentarismo proviene del latín sedentarius, de sedere, estar sentado. En el 2002, la Organización Mundial de la Salud definió el sedentarismo como poca agitación o movimiento (15). Desde la perspectiva del gasto de energía, una persona es considerada como sedentaria cuando en promedio las actividades cotidianas no generan un consumo superior al $10 \%$ con respecto al reposo. Como unidad de medida para el gasto de energía se ha establecido al equivalente metabólico MET, por sus siglas en inglés (16).

Un individuo sedentario es aquel cuyo gasto energético es menor a 150 kilocalorías/día. Actividades de intensidad moderada asociadas a beneficios en salud generan un gasto de 3 a 4 equivalentes metabólicos. Esto quiere decir que una persona sedentaria participa en actividades físicas por periodos menores de $20 \mathrm{minu}$ tos diarios, con una frecuencia menor de tres veces por semana. Asimismo, es posible determinar el nivel de sedentarismo por medio de la cantidad de pasos diarios que acumula una persona. Una persona se considera activa cuando logra acumular un total de 10000 pasos por día.

El comportamiento sedentario se ha convertido en un tema cultural y social. Si bien, el sedentarismo es una problemática global, existe una clara diferencia entre las culturas occidentales y orientales; en estas últimas, pese al avance tecnológico y al desarrollo urbanístico, los niveles de actividad física aún son altos y se evidencia una incorporación del movimiento en actividad destinada al desplazamiento y al tiempo libre.

En los países con mayor producto interno bruto, casi un tercio de la población adulta cumple con las recomendaciones mundiales de actividad física; por su parte, los dos tercios restantes se consideran personas sedentarias (17).

La Organización Panamericana de la Salud (OPS) y la OMS, afirman que en 
América Latina, cerca de tres cuartos de la población tiene un estilo de vida sedentario. Esto quiere decir que gran parte de la población en todas las edades es físicamente inactiva (18).

En las bases de datos especializadas, reposa una amplia cantidad de investigaciones relacionadas con el sedentarismo. Durante mucho tiempo su conceptualización y forma de medición fue muy variada, lo cual ha afectado la universalización de los resultados. De manera reciente, se ha logrado identificar que el sedentarismo puede ser medido a través de encuestas, escalas, formularios y hasta por medio de llamadas telefónicas que permiten cuantificar los tiempos destinados a actividades de bajo gasto energético a nivel individual y colectivo (19).

\section{Metodología}

Mediante el estudio descriptivo transversal y de alcance descriptivo, se determinará la prevalencia de sedentarismo y otros factores asociados. Para la recolección de la información se utilizará la versión larga del Cuestionario Internacional de Actividad Física (IPAQ, por sus siglas en inglés). Además, se recolectará información relacionada con variables sociodemográficas de los encuestados y variables antropométricas como peso y talla, las cuales se registraron siguiendo el protocolo de la Sociedad para el Desarrollo de la Cineantropometría (ISAK, por sus siglas en inglés). Estas dos variables serán medidas en báscula marca Seca 876 y estadiómetro marca Seca
217, respectivamente. Como universo poblacional, se consideró a la población entre 18 a 64 años residente en el área urbana del municipio de Pereira y que al momento de la encuesta no esté en estado de embarazo.

Considerando que los sujetos objeto de esta investigación no tienen ubicación fija, se definió como unidad de muestreo el hogar. Tomando como base la información catastral se estimó una población $\mathrm{N}=74025$. A partir de este valor, se estimó la muestra $\mathrm{n}=950$ con un error permitido del 0,5 . La selección de los hogares a encuestar se desarrollará de manera aleatoria a partir de la información catastral y se empleó una técnica de selección por georreferenciación.

\section{Resultados esperados}

- Se logra un diseño metodológico ajustado a los lineamientos bioestadísticos para el desarrollo de un estudio de prevalencia a nivel ciudad.

- Se logra estimar una muestra representativa para la ciudad de Pereira.

- Se establece un corpus de información adecuado para el diseño y desarrollo del estudio.

\section{Impactos}

Se espera construir una información útil para que la autoridad territorial establezca los indicadores de salud pública, que permitan monitorear los resultados de los programas de promoción de la actividad física, a la vez que se fortalezca el sistema de vigilancia epidemiológica de la ciudad. 


\section{Referencias}

1. Caspersen CJ, Christenson GM. Physical activity, exercise, and physical fitness: Definitions and distinctions for health-related research. Public Health Rep. 1985; 100(2):126-131.

2. Departamento administrativo de recreación y deporte. Tomo ॥. Fundamentos para hábitos de vida saludable. 2011.

3. Vidarte-Claros J, Vélez-Álvarez C, Parra-Sánchez J. El nivel de sedentarismo en nueve ciudades colombianas: análisis de clúster. Arch Med Deport. 2015; 33(4):253-257.

4. Pietiläinen $\mathrm{KH}$, Kaprio J, Borg P, Plasqui $\mathrm{G}$, Yki-Järvinen $\mathrm{H}$, Kujala UM, et al. Physical inactivity and obesity: A vicious circle. Obesity [Internet]. 2008; Feb [cited 2020 May 14];16(2):409-414. http://doi.wiley.com/10.1038/oby.2007.72

5. Knight JA. Physical inactivity: Associated diseases and disorders. Ann Clin Lab Sci. 2012; Jun 20;42(3):320-337.

6. World Health Organization. Global Health Risks [Internet]. 2009. http://www. who.int/healthinfo/global_burden_disease/GlobalHealthRisks_report_ful I.pdf

7. Ding D, Lawson KD, Kolbe-Alexander TL, Finkelstein EA, Katzmarzyk PT, van Mechelen W, et al. The economic burden of physical inactivity: A global analysis of major non-communicable diseases. Lancet [Internet]. 2016; 388(10051):1311$1324 . \quad$ http://dx.doi.org/10.1016/ s0140-6736(16)30383-X

8. Bailey DP, Orton CJ, Maylor BD, Zakrzewski-fruer JK. Cardiometabolic response to a single high-intensity interval exercise session versus breaking up sedentary time with fragmented high-intensity interval exercise authors. Physiol. 2019.

220 9. Lavie CJ, Ozemek C, Carbone S, Katzmarzyk PT, Blair SN. Sedentary behavior, exercise, and cardiovascular health. 2019 [cited 2020 Jan 24]. http://ahajournals.org

10. Álvarez CV, Claros JAV, Ocampo DMR, Martínez APM. Prevalencia de actividad física y factores relacionados en la población de 18-60 años - Pereira 2010. Rev Médica Risaralda [Internet]. 2011; 17(2):85-90. http://revistas.utp. edu.co/index.php/revistamedica/article/ view/7589/4511

11. Van der Ploeg HP. Sitting time and all-cause mortality risk in 222497 Australian adults. Arch Intern Med [Internet]. 2012; Mar 26 [cited 2020 May 12]; 172(6):494. http://archinte.jamanetwork.com/article.aspx?doi=10.1001/ archinternmed.2011.2174

12. Rojas-Valencia A, Marín-Buitrago A. Espacio público y calidad de vida en la ciudad de Pereira [Internet]. Pereira. 2009 [cited 2019 Sep 8]. http://repositorio.unilibrepereira.edu.co:8080/pereira/bitstream/ handle/123456789/68 6/ESPACIO PÚBLICO.pdf?sequence $=1$

13. Vélez C, Vidarte JA, Cerón JD. Determinantes sociales de la salud y discapacidad: caso Santiago de Cali. Rev Fac Nac Salud Pública [Internet]. 2013 [cited 2019 Oct 13]; 31:192-202. https://www.redalyc. org/pdf/120/12028727019.pdf

14. ICBF. Encuesta Nacional de la Situación Nutricional. 2015; 1-58. http://www. culturarecreacionydeporte.gov.co/sites/ default/files/2015_diseno- general-ensin_.pdf

15. Familiar I colombiano de B. Encuesta Nacional de Situación Nutricional. Bogotá; 2010.

16. Bernstein MS, Morabia A, Sloutskis D. Definition and prevalence of sedentarism in an urban population. Am J Public Health. $1999 ; 89(6): 862-867$.

17. World Health Organization. Inactividad física: un problema de salud pública mundial. WHO. 2013. 
18. García-Sánchez L, Bonilla-Briceño J, Durán D. Muévase contra el sedentarismo. Facultad de Rehabilitación y DesarroIlo Humano [Internet]. [cited 2020 Jun 18]. (07). www.urosario.edu.co/investigacion

19. Buhring BK, Oliva MP, Bravo CC. Not experimental determination of sedentary behavior in school-age children. Rev Chil Nutr. 2009; 36(1):23-30. 